OPEN ACCESS

Edited by:

Stefano Fiorucci,

University of Perugia, Italy

Reviewed by:

Rinaldo Pellicano,

Molinette Hospital, Italy

Rosanna Villani,

University of Foggia, Italy

${ }^{*}$ Correspondence:

Marco Sanduzzi-Zamparelli msanduzzizamparelli@gmail.com

msanduzzi@clinic.cat

Received: 29 November 2021

Accepted: 03 January 2022

Published: 02 February 2022

Citation:

Cossiga V, Sanduzzi-Zamparelli M.

Sapena V, Guarino M, Dallio M,

Torrisi E, Pignata L, Federico A,

Salomone $F$ and Morisco $F$ (2022)

Beneficial Effects of Silybin Treatment After Viral Eradication in Patients With HCV-Related Advanced Chronic Liver

Disease: A Pilot Study.

Front. Pharmacol. 13:824879.

doi: 10.3389/fphar.2022.824879

\section{Beneficial Effects of Silybin Treatment After Viral Eradication in Patients With HCV-Related Advanced Chronic Liver Disease: A Pilot Study}

\author{
Valentina Cossiga ${ }^{1}$, Marco Sanduzzi-Zamparelli ${ }^{1 *}$, Victor Sapena ${ }^{2}$, Maria Guarino ${ }^{1}$, \\ Marcello Dallio ${ }^{3}$, Emanuele Torrisi ${ }^{4}$, Luca Pignata ${ }^{1}$, Alessandro Federico ${ }^{3}$, \\ Federico Salomone ${ }^{4}$ and Filomena Morisco ${ }^{1}$
}

${ }^{1}$ Department of Clinical Medicine and Surgery, Gastroenterology and Hepatology Unit, University of Naples "Federico II", Naples, Italy, ${ }^{2}$ Medical Statistics Core Facility, Institut D'Investigacions Biomédiques August Pi i Sunyer (IDIBAPS), Hospital Clinic Barcelona, Barcelona, Spain, ${ }^{3}$ Department of Precision Medicine, Hepato-Gastroenterology Unit, University of Campania "Luigi Vanvitelli", Naples, Italy, ${ }^{4}$ Division of Gastroenterology, Acireale Hospital, Azienda Sanitaria Provinciale di Catania, Catania, Italy

Introduction and Aims: HCV eradication by direct-acting antivirals (DAAs) improves liver outcomes and reduces overall liver mortality. However, patients with advanced chronic liver disease (ACLD) may experience a progression of liver disease despite viral clearance. Silybin has shown hepatoprotective effects in experimental models, but clinical data are limited. The aim of this study is to evaluate the effect of a highly bioavailable form of silybin on liver fibrosis in patients with HCV-related ACLD after viral eradication with DAAs, in comparison with the standard of care.

Methods: In this multicenter and prospective study, HCV patients with ACLD achieving SVR12 were treated with the combination of silybinphospholipid complex with vitamin D and vitamin $\mathrm{E}$ (Realsil $100 \mathrm{D}^{\circledR}$, Ibi Lorenzini S.p.A., Aprilia, Italy) for 12 months (R group) compared to controls (C group). Patients were submitted to transient elastography (TE) and to the enhanced liver fibrosis (ELF) test at baseline, week 24, and week 48.

Results: One hundred sixteen patients were enrolled, 56 in the R group and 60 in the C group. The median age was 68 years, and $53 \%$ were male, with no differences between groups. In both groups, liver stiffness improved at 6 and 12 months compared to baseline. However, patients in the $\mathrm{R}$ group compared to those in the $\mathrm{C}$ group showed a higher reduction of liver stiffness after 6 months $(-2.05,95 \% \mathrm{Cl}-3.89$ to $-0.22, p<0.05)$ and 12 months of treatment $(-2.79,95 \% \mathrm{Cl}-4.5$ to $-1.09, p<0.01)$ in comparison with baseline. No significant difference in the reduction of ELF was observed between the two groups. During the follow-up, four patients developed HCC, all in the C group.

Conclusions: In HCV-related ACLD, the hepatoprotective effects of silybin may represent a tool to counteract liver disease progression.

Keywords: HCV, DAA, silybin, transient elastography, advanced liver disease 


\section{INTRODUCTION}

More than 180 million people worldwide are infected with hepatitis $\mathrm{C}$ virus $(\mathrm{HCV})$, which still remains a major cause of chronic liver disease (Thrift et al., 2017). Over the last few years, direct-acting antivirals (DAAs) became available for $\mathrm{HCV}$ treatment, with high rates ( $>95 \%)$ of sustained virological response (SVR), regardless of the stage of fibrosis and viral genotype (Ponziani et al., 2017). Notably, SVR achievement is associated with improvement of liver function, regression of fibrosis, reduction of portal hypertension, and an overall decrease of liver-related events and mortality (Innes et al., 2017; Carrat et al., 2019; Morisco et al., 2021). However, patients with advanced chronic liver disease (ACLD) may experience a progression of liver disease despite viral clearance (Carrat et al., 2019). For this reason, the assessment of liver fibrosis prior to and after antiviral treatment is fundamental to determining the prognosis and establishing an adequate followup (Singal et al., 2010). The most widely used and validated technique, in this setting of patients, is transient elastography (TE) which is the non-invasive method with highest accuracy to identify the advanced stage of fibrosis (European Association for the Study of the Liver 2021).

Silymarin is the seed extract of milk thistle (Silybum marianum) consisting of seven flavonolignans and taxifolin, which is widely used in a variety of chronic liver diseases for its anti-inflammatory, anti-fibrotic, and antioxidant effects (Federico et al., 2017). Studies in experimental models of liver disease have shown that silybin, the main component of silymarin, may be considered an anti-fibrotic molecule inhibiting transforming growth factor $\beta$ (TGF $\beta)$ and plateletderived growth factor (PDGF) cascades (Muriel et al., 2005). Besides, it inhibits the pro-inflammatory signals involved in the synthesis of cytokines such as tumor necrosis factor $\alpha$ (TNF- $\alpha$ ) and in the modulation of apoptosis modulating levels of bcl2-like protein-4 (Bax), while the antioxidant effect is mainly related to its ability to act as a free radical scavenging and lipid peroxidation inhibitor (Loguercio and Festi, 2011; Salomone et al., 2016; Abenavoli et al., 2018). Furthermore, clinical studies suggest that the oral administration of silybin in patients with liver diseases is able to reduce hepatic inflammation translating in a reduction of serum transaminases with an excellent safety profile (LozanoSepulveda et al., 2015).

In this scenario, the aim of the present study is to evaluate the effects of a combination of silybinphospholipid complex with vitamin D and vitamin E (Realsil 100D ${ }^{*}$, Ibi Lorenzini S.p.A., Aprilia, Italy) on the modulation of liver fibrosis in comparison with the control group of patients with HCV-related ACLD achieving SVR after DAA treatment.

\section{MATERIALS AND METHODS}

\section{Patients and Study Design}

This is a multicenter, prospective, controlled, and interventional study assessing the effects of an oral combination of silybinphospholipid complex with vitamin $\mathrm{D}$ and vitamin $\mathrm{E}$ (Realsil $100 D^{\circledast}$, Ibi Lorenzini S.p.A., Aprilia, Italy) in patients with HCV-related ACLD who achieved SVR after DAAs. The study was conducted in three centers in Southern Italy (Department of Clinical Medicine and Surgery of Federico II University of Naples, Division of Gastroenterology of Acireale Hospital, and Hepato-Gastroenterology Unit of University of Campania "Luigi Vanvitelli"). The ethics committee of Federico II University approved the study protocol (no. 34/ 2016), and all patients signed an informed written consent form.

Inclusion criteria for the study were 1) severe fibrosis, before starting antiviral treatment, defined by liver stiffness measurement (LSM) $\geq 9.5 \mathrm{kPa} \quad$ (corresponding to $\geq \mathrm{F} 3$ according to the METAVIR score) and/or radiological/clinical signs of cirrhosis and 2) confirmed SVR at 12 weeks after the end of antiviral treatment. Exclusion criteria were 1) Child-Pugh score $>6$ points; 2 ) active or previous history of hepatocellular carcinoma (HCC); 3) prior liver transplantation; 4) concomitant coinfection with hepatitis B virus (HBV) and/or human immunodeficiency virus (HIV); 5) other causes of liver disease; 6) decompensated diabetes mellitus; or 7) body mass index $(\mathrm{BMI})>30$.

Between January 2016 and December 2017, 120 patients were enrolled according to inclusion and exclusion criteria. Sixty patients were randomized in the $R$ group and 60 in the $C$ group. Four patients in the $\mathrm{R}$ group withdrew the informed consent before starting the treatment, and 116 patients were considered for the final analysis (56 in the R group and 60 in the $\mathrm{C}$ group). A study flowchart is depicted in Figure 1.

At baseline (SVR12), patients in the $\mathrm{R}$ group received active treatment with Realsil 100D ${ }^{\circledR}$ (303 mg of silybinphospholipid complex, $10 \mu \mathrm{g}$ of vitamin $\mathrm{D}$, and $15 \mathrm{mg}$ of vitamin $\mathrm{E}$ ) twice a day for 12 months. In the control group (C), patients underwent no intervention and continued standard followup after SVR.

Demographic and clinical data (age, gender, body mass index (BMI), diabetes mellitus, arterial hypertension, history of previous cancer), HCV genotype, type of DAA therapy, and laboratory parameters (platelet count, transaminases) were collected at study inclusion. SVR was defined as undetectable HCV RNA at 12 weeks after the end of treatment. Laboratory and clinical parameters were repeated at 6 and 12 months after the enrollment. Liver-related events were collected until death, until withdrawal of informed consent, or until November 20, 2020.

\section{Non-Invasive Assessment of Liver Fibrosis}

Liver fibrosis was evaluated non-invasively with both TE and enhanced liver fibrosis (ELF) test at baseline (SVR12) and at 6 and 12 months. Patients were submitted to TE and to a fasting blood sample in the same day. Liver stiffness measurements (LSMs) were performed by a single well-trained operator in each center using FibroScan ${ }^{\circledR} 502$ Touch (EchoSens, Paris, France). The results were expressed in kilopascal $(\mathrm{kPa})$ in the range from 2.5 to $75 \mathrm{kPa}$. The IQR was defined as an index of intrinsic variability of LSM. Only those measurements with more than ten successful acquisitions, with a success rate of at least $60 \%$ and an interquartile range lower than $30 \%$, were classified as valid and 


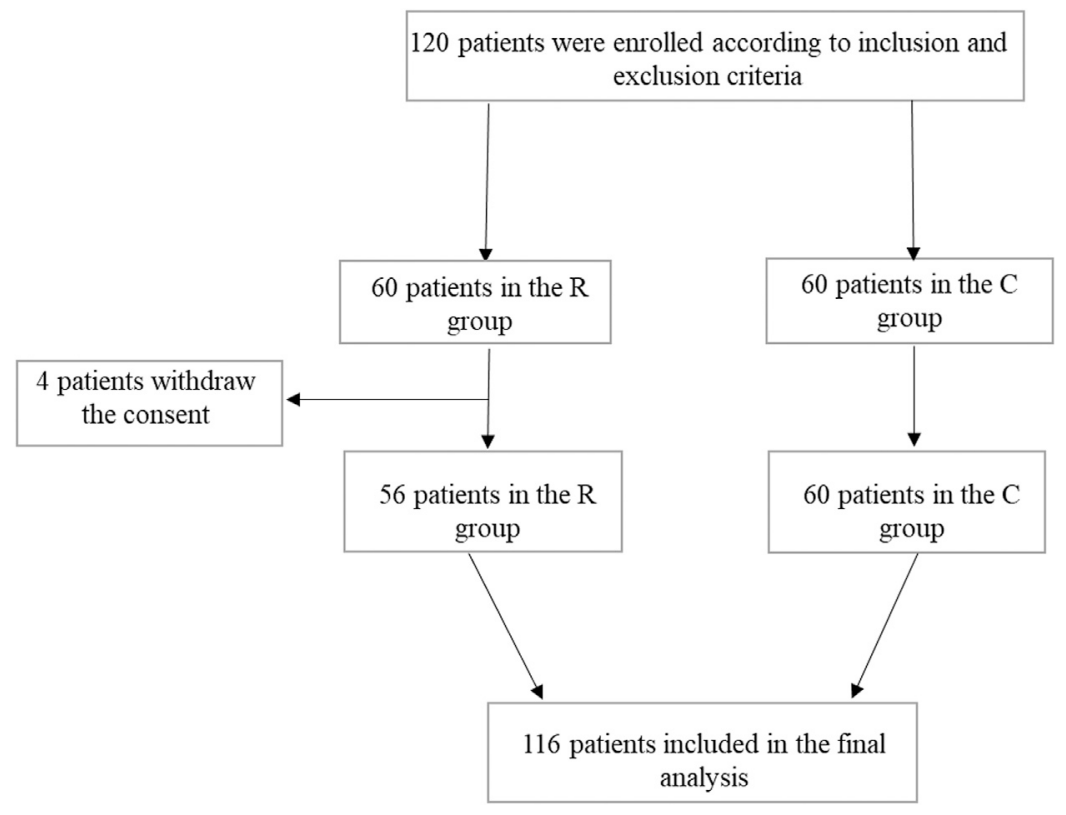

FIGURE 1 | Study flowchart.

taken into consideration for statistical evaluation (Nitta et al., 2009).

For the ELF test, fasting blood samples were obtained in the same day of LSM. All sera were frozen and stored at $-20^{\circ} \mathrm{C}$ until determination. Samples were assayed in an automated analyzer that performs magnetic separation enzyme immunoassay tests (ADVIA Centaur; Siemens Healthcare Diagnostics, Tarrytown, NY). The results were entered into the manufacturer's published algorithm to derive an ELF score $[E L F=2.278+0.851 \ln (\mathrm{HA})+$ $0.751 \ln (\mathrm{PIIINP})+0.394 \ln (\mathrm{TIMP}-1)]$.

\section{Statistical Analysis}

Quantitative variables were expressed as median and interquartile range [IQR 25th-75th percentiles]. Categorical variables were described as absolute frequencies and percentages (\%).Fisher's exact test was used to compare categorical variables, and for quantitative or ordinal variables, we used non-parametric methods: Mann-Whitney (two groups) for independent data and Wilcoxon signed-rank test (two groups) for dependent data.

Liver stiffness variations between treatment and placebo groups were estimated, compared between baseline (SVR12) and the end of treatment, by multivariable linear mixed models for repeated measurements (MMRMs), using age, gender, cirrhosis, ALT, and AST as adjusting factors. The reported $p$-values for MMRMs were calculated with a nonparametric approach, obtained from the same model but using ranges of liver stiffness instead of raw values.

All statistical analyses were performed using SAS software v9.4 ${ }^{\circledR}$, and we considered a two-sided type I error as $5 \%$.

\section{RESULTS}

Table 1 summarizes the baseline characteristics of the enrolled patients. Overall, 53\% were male, with a median age of 68 [IQR 60-72] years and a median BMI of 25.8 [IQR $23.6-29.1] \mathrm{kg} / \mathrm{m}^{2}$. There were no significant differences between groups regarding age, sex, and BMI.

Overall, the median baseline LSM was 9.6 [IQR 7.2-14] $\mathrm{kPa}$. In particular, the LSM was 10.2 [IQR 7.2-15] $\mathrm{kPa}$ in the $\mathrm{R}$ group and 9.1 [IQR $7.3-12$ ] $\mathrm{kPa}$ in the $\mathrm{C}$ group $(p=0.46)$. Overall, $29.3 \%$ of patients had history of arterial hypertension, and $22.4 \%$ of them had diabetes mellitus. The most frequent HCV genotype was $1(75 \%)$ followed by genotype $2(16.4 \%)$, without difference between groups. The median follow-up was 36.2 (IQR 31.4-41.7) months in the R group and 37.1 (IQR 25.7-46.6) in the C group. All but one patient who withdrew informed consent completed the treatment of 12 months in the R group.

At baseline (SVR12), 102 patients had Child-Pugh (CP) score $\mathrm{A}$ and 3 patients had $\mathrm{CP}$ score $\mathrm{B}$. The variations of $\mathrm{CP}$ score before and after treatment are detailed in Supplementary Table S1.

\section{Transient Elastography and Enhanced Liver Fibrosis Variations}

In both groups, LSM improved at 6 and 12 months in comparison with baseline. The multivariate model, adjusted for age, gender, cirrhosis, and AST and ALT parameters, showed that patients in the $\mathrm{R}$ group compared to those in the $\mathrm{C}$ group had a more 
TABLE 1 | Baseline characteristics of patients at study inclusion.

\begin{tabular}{|c|c|c|c|c|}
\hline & Overall $(n=116)$ & $R$ group $(n=56)$ & C group $(n=60)$ & $p$-Value \\
\hline Age (years), median [IQR] & $68[60-72]$ & $68[59.5-71.5]$ & $67.5[60-73.5]$ & 0.8595 \\
\hline Gender (male), n (\%) & $62(53.4)$ & $30(53.6)$ & $32(53.3)$ & 0.9999 \\
\hline BMI $\left(\mathrm{kg} / \mathrm{m}^{2}\right)$, median $[\mathrm{IQR}]$ & $25.8[23.6-29.1]$ & $25.5[23.7-28.1]$ & $25.9[23.1-27.6]$ & 0.8531 \\
\hline ELF, median $\left[\mathrm{IQR}^{\mathrm{a}}\right.$ & $10.7[9.6-11.2]$ & $10.7[10-11.2]$ & $10.7[9.5-11.2]$ & 0.6120 \\
\hline TE (kPa), median [IQR] & $9.6[7.2-14]$ & $10.2[7.2-15]$ & $9.1[7.3-12]$ & 0.4657 \\
\hline History of AHT (yes), n (\%) & 34 (29.3) & $18(32)$ & $16(26)$ & 0.5462 \\
\hline History of diabetes mellitus (yes), n (\%) & $26(22.4)$ & $12(21)$ & $14(23)$ & 0.8276 \\
\hline Platelets $\left(10^{9}\right)$, median [IQR] & $172[136-211]$ & 159 [135-204] & $174[140-220]$ & 0.3186 \\
\hline ALT (IU/L), median [IQR] & $22[15.5-28]$ & $22[15.5-28]$ & $21[14.2-29]$ & 0.2085 \\
\hline AST (IU/L), median [IQR] & 23 [19-27] & 21 [18-27] & 23 [19-29] & 0.1197 \\
\hline HCV-RNA before DAA $\left(10^{6} \mathrm{IU} / \mathrm{ml}\right)$ & $1.7[0.8-3.3]$ & $1.6[0.9-3.5]$ & $1.7[0.5-3.1]$ & 0.5932 \\
\hline HCV genotype, n (\%) & - & - & - & 0.3325 \\
\hline 1 & $87(75)$ & $42(75)$ & $45(75)$ & - \\
\hline 2 & $19(16.4)$ & $7(12.5)$ & $12(20)$ & - \\
\hline 3 & $8(6.9)$ & $5(8.9)$ & $3(5)$ & - \\
\hline 4 & $2(1.7)$ & $2(3.6)$ & 0 & - \\
\hline
\end{tabular}

BMI: body mass index; ELF: enhanced liver fibrosis; TE: transient elastography; AHT: arterial hypertension; ALT: alanine aminotransferase; AST: aspartate aminotransferase; HCV: hepatitis $C$ virus.

${ }^{a}$ Of the whole cohort, in $49 \%$ of the cases, the ELF score was not calculable.

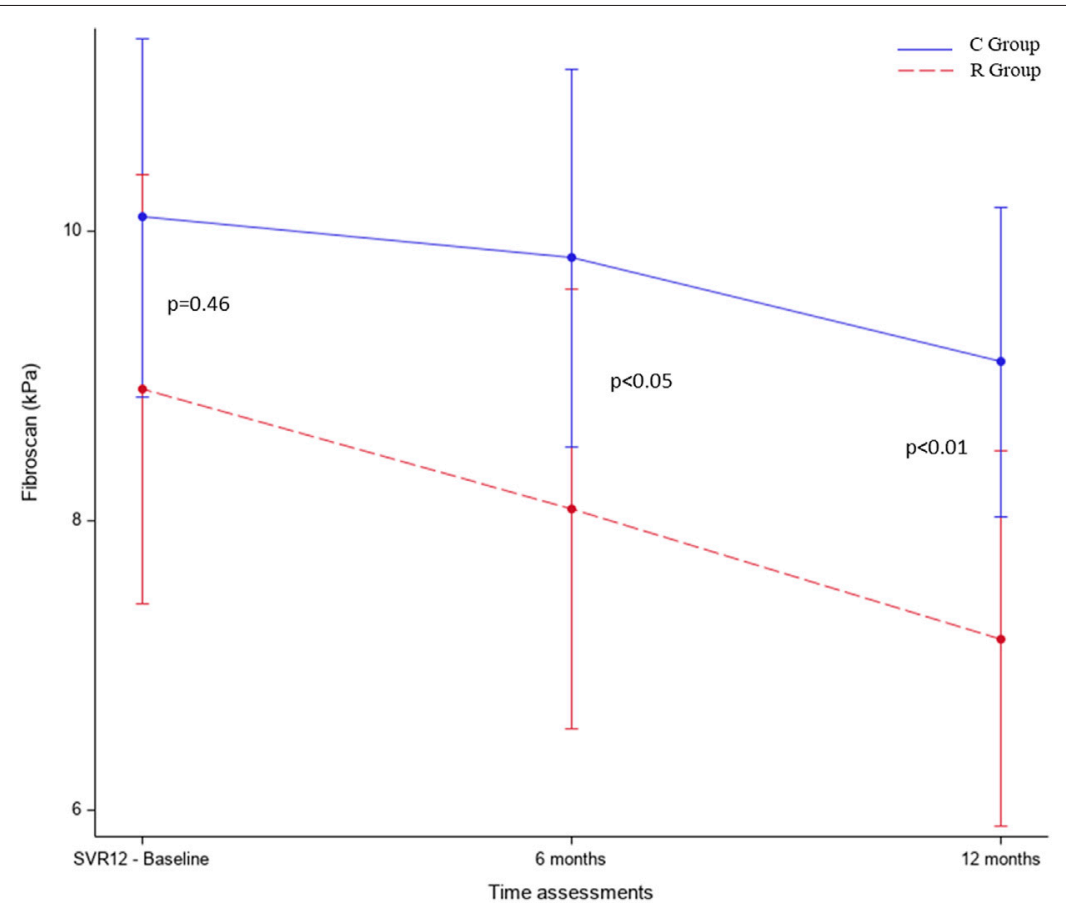

FIGURE 2 | Liver stiffness variations in both groups between baseline and end of the study in the multivariate model adjusted for age, gender, cirrhosis, ALT, and AST. R group: patients who received active treatment; C group: patients with no intervention.

significant improvement of LSM both after $6(-2.05,95 \% \mathrm{CI}$ -3.89 to $-0.22, p<0.05)$ and 12 months of treatment $(-2.79,95 \%$ $\mathrm{CI}-4.5$ to $-1.09, p<0.01$ ) in comparison with baseline (Figure 2 and Table 2). Contrarily, no significant changes were observed in the ELF score after 12 months in the R group compared to the $\mathrm{C}$ group (ELF24-ELF12 diff $=0.4 \mathrm{kPa}, p=0.227$ ) (Table 3 ).

\section{Safety and Long-Term Outcome}

No patient presented treatment-related adverse events. During the extended follow-up, no patients developed HCC in the R group, while the occurrence of HCC was observed in four patients of the C group (all patients with LSM $>14 \mathrm{kPa}$, corresponding to F4 according to the METAVIR score). No case of liver 
TABLE 2 | Results of multivariate MMRMs adjusted by age, gender, cirrhosis, and ALT and AST parameters. Estimated mean differences, and their 95\% Cl, between treatments and visits by MMRMs for transient elastography. The multivariate model includes treatment, visit, treatment and visit interaction, age, gender, the presence of cirrhosis, ALT, and AST. The result of MMRM analysis corresponds to the difference of the changes in transient elastography between the two arms at different time points.

\begin{tabular}{|c|c|c|c|c|c|}
\hline Time point 1 & Time point 2 & Treatment & Treatment (ref.) & $\begin{array}{c}\text { Difference in } \\
\text { transient elastography } \\
(95 \% \mathrm{Cl})\end{array}$ & $p$-Value ${ }^{a}$ \\
\hline Baseline & Baseline & $\mathrm{R}$ group & C group & $-1.19(-3.03--0.66)$ & 0.2143 \\
\hline $6 m$ & $6 m$ & $R$ group & C group & $-1.74(-3.65--0.18)$ & 0.2578 \\
\hline $6 m$ & Baseline & R group & C group & $-2.02(-3.89---0.15)$ & 0.0258 \\
\hline $12 \mathrm{~m}$ & $12 \mathrm{~m}$ & $R$ group & C group & $-1.91(-3.48---0.34)$ & 0.0203 \\
\hline $12 \mathrm{~m}$ & $6 m$ & $R$ group & C group & $-2.63(-4.38---0.89)$ & 0.0122 \\
\hline $12 \mathrm{~m}$ & Baseline & $R$ group & C group & $-2.91(-4.61---1.21)$ & 0.0003 \\
\hline
\end{tabular}

${ }^{a}$ Non-parametric test; $6 \mathrm{~m}$ : 6 months (24 weeks); 12m: 12 months (48 weeks).

MMRM: multivariable linear mixed model of repeated measurements.

TABLE 3 | ELF variations at baseline and 12 months after treatment.

\begin{tabular}{|c|c|c|c|c|}
\hline & \multicolumn{2}{|c|}{ SVR12 } & \multicolumn{2}{|c|}{12 months } \\
\hline & $\mathbf{R}$ group & C group & $\mathbf{R}$ group & C group \\
\hline ELF, median [IQR] & 10.7 [10-11.2] & $10.7[9.5-11.2]$ & $9.9[9.2-11.1]$ & $10.1[9.3-10.8]$ \\
\hline
\end{tabular}

decompensation neither variceal bleeding was reported in both groups. Four deaths were reported ( 2 in the R group and 2 in the $\mathrm{C}$ group) and were due to non-liver-related deaths in two patients.

\section{DISCUSSION}

The advent of DAA-based regimens for HCV infection, characterized by excellent efficacy and safety profiles, made possible HCV eradication also in patients with ACLD. Many studies showed that SVR achievement is clearly associated with the improvement of natural history and the reduction of liverrelated events and mortality (D'Ambrosio et al., 2021; Di Marco et al., 2016; Van der Meer et al., 2017; Nahon et al., 2017). However, the issues regarding regression of liver fibrosis and portal hypertension remain controversial, especially in patients with cirrhosis and clinically significant portal hypertension (CSPH) (Rosso et al., 2020). Thus, the synergic action of additional molecules with anti-fibrotic effect, such as silybin, could have a relevant role.

In this multicenter, prospective, and interventional study, patients orally treated with a highly bioavailable form of silybin showed a more significant improvement of liver stiffness at 6 and 12 months compared to patients receiving no intervention after achieving SVR with DAAs. These preliminary results underline the plausible antifibrotic effect of silybin with consequent reduction of liver damage through the softening of inflammatory cascade and immune system modulation (Salomone et al., 2016). Moreover, silymarin seems to effectively interfere with fibrogenesis at different levels of the process. In a mouse model of CCl4-induced liver fibrosis, Clichici et al. (2015) demonstrated that the administration of silymarin for 4 weeks, at doses usually used in clinical practice as adjuvant in hypertransaminasemia, favors the reduction of hepatocyte damage, oxidative stress markers, and fibrosis score and the activation of both hepatic stellate cells (HSCs) and Kupffer cells.

Another anti-fibrotic effect of silybin is the antagonization of platelet-activating factor (PAF) action. Indeed, the PAF stimulates HSCs to produce a large quantity of collagen ( $\mathrm{Lu}$ et al., 2008). This capacity of PAF would be opposed by the process of acetylation supported by lysophosphatidylcholine acyltransferase (LPCAT) enzymes, whose expression is clearly lower in cirrhotic patients compared to controls. In this regard, silybin is able to antagonize the profibrotic effect of PAF through the increase of LPCAT expression, as well as through a direct reduction of PAF in cirrhotic Wistar rats (Stanca et al., 2013). Moreover, in a study on rats, treatment with silybin reduced the production of collagen-I and $\alpha$-SMA (alpha-smooth muscle actin), with consequent inhibition of HSC activation ( $\mathrm{Hu}$ et al., 2019). Of note, it has been demonstrated that silybin is able to reduce liver fibrosis in NAFLD patients with paired liver biopsy before and after treatment (Loguercio et al., 2012).

In our cohort, with a median follow-up of 23 months, four patients developed HCC, all in the control group. This finding could be further analyzed in a larger cohort of patients considering the potential anti-cancer modulatory effect of silybin. Indeed, silybin could interfere with the tumoral process through inflammatory cascade regulation and by decreasing the ROS genotoxic potential. Moreover, it could block most of the signaling pathways activated in HCC (Gopalakrishnan et al., 2013; Mao et al., 2018).

The present study has some limitations. First, the assessment of LSM with transient elastography after DAA therapy carries a lower reproducibility in comparison with its pre-DAA value (European Association for the Study of the Liver 2021). Moreover, this is a pilot study with a small number of patients, and especially, the long-term outcomes should be further assessed in larger studies to confirm these results. 
In conclusion, in our study, we showed that the combination of silybinphospholipid complex with vitamin D and vitamin E (Realsil $100 D^{\otimes}$, Ibi Lorenzini S.p.A., Aprilia, Italy) is potentially able to favor the regression of liver fibrosis in patients with SVR after DAA treatment for HCV infection. Larger studies are needed to carefully evaluate how this could potentially impact on liverrelated outcomes.

\section{DATA AVAILABILITY STATEMENT}

The raw data supporting the conclusions of this article will be made available by the authors, without undue reservation.

\section{ETHICS STATEMENT}

The studies involving human participants were reviewed and approved by Comitato Etico Federico II-University of Naples Federico II-Via Sergio Pansini 5, 80131 Naples, Italy. The patients/participants provided their written informed consent to participate in this study.

\section{REFERENCES}

Abenavoli, L., Izzo, A. A., Milić, N., Cicala, C., Santini, A., and Capasso, R. (2018). Milk Thistle (Silybum marianum): A Concise Overview on its Chemistry, Pharmacological, and Nutraceutical Uses in Liver Diseases. Phytother Res. 32, 2202-2213. doi:10.1002/ptr.6171

Carrat, F., Fontaine, H., Dorival, C., Simony, M., Diallo, A., Hezode, C., et al. (2019). Clinical Outcomes in Patients with Chronic Hepatitis C after DirectActing Antiviral Treatment: a Prospective Cohort Study. Lancet 393, 1453-1464. doi:10.1016/S0140-6736(18)32111-1

Clichici, S., Olteanu, D., Nagy, A. L., Oros, A., Filip, A., and Mircea, P. A. (2015). Silymarin Inhibits the Progression of Fibrosis in the Early Stages of Liver Injury in $\mathrm{CCl}_{4}$-Treated Rats. J. Med. Food 18, 290-298. doi:10.1089/jmf.2013.0179

D’Ambrosio, R., Degasperi, E., Anolli, M. P., Fanetti, I., Borghi, M., Soffredini, R., et al. (2021). Incidence of Liver- and Non-liver-related Outcomes in Patients with HCV-Cirrhosis after SVR. J. Hepatol. S0168-8278 (21), 02043. doi:10. 1016/j.jhep.2021.09.013

Di Marco, V., Calvaruso, V., Ferraro, D., Bavetta, M. G., Cabibbo, G., Conte, E., et al. (2016). Effects of Eradicating Hepatitis C Virus Infection in Patients with Cirrhosis Differ with Stage of portal Hypertension. Gastroenterology 151, 130-e2. doi:10.1053/j.gastro.2016.03.036

European Association for the Study of the Liver (2021). EASL Clinical Practice Guidelines on Non-invasive Tests for Evaluation of Liver Disease Severity and Prognosis - 2021 Update. J. Hepatol. 75, 659-689. doi:10.1016/j.jhep.2021.05.025

Federico, A., Dallio, M., and Loguercio, C. (2017). Silymarin/Silybin and Chronic Liver Disease: A Marriage of Many Years. Molecules 22, 191. doi:10.3390/ molecules22020191

Gopalakrishnan, R., Sundaram, J., Sattu, K., Pandi, A., and Thiruvengadam, D. (2013). Dietary Supplementation of Silymarin Is Associated with Decreased Cell Proliferation, Increased Apoptosis, and Activation of Detoxification System in Hepatocellular Carcinoma. Mol. Cel. Biochem. 377, 163-176. doi:10.1007/s11010-013-1582-1

Hu, R., Jia, W. Y., Xu, S. F., Zhu, Z. W., Xiao, Z., Yu, S. Y., et al. (2019). Xiaochaihutang Inhibits the Activation of Hepatic Stellate Cell Line T6 through the Nrf2 Pathway. Front. Pharmacol. 9, 1516. doi:10.3389/fphar.2018.01516

Innes, H., McDonald, S., Hayes, P., Dillon, J. F., Allen, S., Goldberg, D., et al. (2017). Mortality in Hepatitis C Patients Who Achieve a Sustained Viral Response Compared to the General Population. J. Hepatol. 66, 19-27. doi:10.1016/j.jhep. 2016.08.004

Loguercio, C., Andreone, P., Brisc, C., Brisc, M. C., Bugianesi, E., Chiaramonte, M., et al. (2012). Silybin Combined with Phosphatidylcholine and Vitamin E in

\section{AUTHOR CONTRIBUTIONS}

VC, MS-Z, and FM designed the research. MG, MD, EM, and LP conducted the research. VS analyzed the data. VC and MS-Z wrote the paper. AF, FS, and FM critically revised the manuscript. All authors read and approved the final manuscript.

\section{FUNDING}

This work was supported by a non-conditioning contribution by Ibi Lorenzini S.p.A., Italy.

\section{SUPPLEMENTARY MATERIAL}

The Supplementary Material for this article can be found online at: https://www.frontiersin.org/articles/10.3389/fphar.2022.824879/ full\#supplementary-material

Patients with Nonalcoholic Fatty Liver Disease: a Randomized Controlled Trial. Free Radic. Biol. Med. 52, 1658-1665. doi:10.1016/j.freeradbiomed.2012.02.008 Loguercio, C., and Festi, D. (2011). Silybin and the Liver: From Basic Research to Clinical Practice. World J. Gastroenterol. 17, 2288-2301. doi:10.3748/wjg.v17.i18.2288

Lozano-Sepulveda, S. A., Bryan-Marrugo, O. L., Cordova-Fletes, C., Gutierrez-Ruiz, M. C., and Rivas-Estilla, A. M. (2015). Oxidative Stress Modulation in Hepatitis C Virus Infected Cells. World J. Hepatol. 7, 2880-2889. doi:10.4254/wjh.v7.i29.2880

Lu, Y. Y., Wang, C. P., Zhou, L., Chen, Y., Su, S. H., Feng, Y. Y., et al. (2008). Synthesis of Platelet-Activating Factor and its Receptor Expression in Kupffer Cells in Rat Carbon Tetrachloride-Induced Cirrhosis. World J. Gastroenterol. 14, 764-770. doi:10.3748/wjg.14.764

Mao, J., Yang, H., Cui, T., Pan, P., Kabir, N., Chen, D., et al. (2018). Combined Treatment with Sorafenib and Silibinin Synergistically Targets Both HCC Cells and Cancer Stem Cells by Enhanced Inhibition of the Phosphorylation of STAT3/ERK/AKT. Eur. J. Pharmacol. 832, 39-49. doi:10.1016/j.ejphar.2018.05.027

Morisco, F., Federico, A., Marignani, M., Cannavò, M., Pontillo, G., Guarino, M., et al. (2021). Risk Factors for Liver Decompensation and HCC in HCVCirrhotic Patients after DAAs: A Multicenter Prospective Study. Cancers (Basel) 13 (15), 3810. doi:10.3390/cancers13153810

Muriel, P., Moreno, M. G., Hernández, M. C., Chávez, E., and Alcantar, L. K. (2005). Resolution of Liver Fibrosis in Chronic CCl4 Administration in the Rat after Discontinuation of Treatment: Effect of Silymarin, Silibinin, Colchicine and Trimethylcolchicinic Acid. Basic Clin. Pharmacol. Toxicol. 96, 375-380. doi:10.1111/j.1742-7843.2005.pto_06.x

Nahon, P., Bourcier, V., Layese, R., Audureau, E., Cagnot, C., Marcellin, P., et al. (2017). Eradication of Hepatitis C Virus Infection in Patients with Cirrhosis Reduces Risk of Liver and Non-liver Complications. Gastroenterology 152, 142-e2. doi:10.1053/j.gastro.2016.09.009

Nitta, Y., Kawabe, N., Hashimoto, S., Harata, M., Komura, N., Kobayashi, K., et al. (2009). Liver Stiffness Measured by Transient Elastography Correlates with Fibrosis Area in Liver Biopsy in Patients with Chronic Hepatitis C. Hepatol. Res. 39, 675-684. doi:10.1111/j.1872-034X.2009.00500.x

Ponziani, F. R., Mangiola, F., Binda, C., Zocco, M. A., Siciliano, M., Grieco, A., et al. (2017). Future of Liver Disease in the Era of Direct Acting Antivirals for the Treatment of Hepatitis C. World J. Hepatol. 9, 352-367. doi:10.4254/wjh.v9.i7.352

Rosso, C., Caviglia, G. P., Younes, R., Ribaldone, D. G., Fagoonee, S., Pellicano, R., et al. (2020). Molecular Mechanisms of Hepatic Fibrosis in Chronic Liver Diseases. Minerva Biotecnologica 32, 121-127. doi:10.23736/s1120-4826.20.02619-1

Salomone, F., Godos, J., and Zelber-Sagi, S. (2016). Natural Antioxidants for Nonalcoholic Fatty Liver Disease: Molecular Targets and Clinical Perspectives. Liver Int. 36 (1), 5-20. doi:10.1111/liv.12975 
Singal, A. G., Volk, M. L., Jensen, D., Di Bisceglie, A. M., and Schoenfeld, P. S. (2010). A Sustained Viral Response Is Associated with Reduced LiverRelated Morbidity and Mortality in Patients with Hepatitis C Virus. Clin. Gastroenterol. Hepatol. 8, 280-e1. doi:10.1016/j.cgh.2009.11.018

Stanca, E., Serviddio, G., Bellanti, F., Vendemiale, G., Siculella, L., and Giudetti, A. M. (2013). Down-regulation of LPCAT Expression Increases PlateletActivating Factor Level in Cirrhotic Rat Liver: Potential Antiinflammatory Effect of Silybin. Biochim. Biophys. Acta 1832, 2019-2026. doi:10.1016/j.bbadis. 2013.07.005

Thrift, A. P., El-Serag, H. B., and Kanwal, F. (2017). Global Epidemiology and burden of HCV Infection and HCV-Related Disease. Nat. Rev. Gastroenterol. Hepatol. 14, 122-132. doi:10.1038/nrgastro.2016.176

Van der Meer, A. J., Feld, J. J., Hofer, H., Almasio, P. L., Calvaruso, V., Fernández-Rodríguez, C. M., et al. (2017). Risk of Cirrhosis-Related Complications in Patients with Advanced Fibrosis Following Hepatitis C Virus Eradication. J. Hepatol. 66, 485-493. doi:10.1016/j.jhep.2016. 10.017

Conflict of Interest: MS-Z Travel grant from Bayer and Eisai; lecture fees form Bayer and BTG.
The authors declare that the research was conducted in the absence of any commercial or financial relationships that could be construed as a potential conflict of interest.

The reviewer RV declared a past co-authorship with the authors MSZ, CS, MG, $\mathrm{MD}, \mathrm{AF}$ to the handling editor.

Publisher's Note: All claims expressed in this article are solely those of the authors and do not necessarily represent those of their affiliated organizations, or those of the publisher, the editors and the reviewers. Any product that may be evaluated in this article, or claim that may be made by its manufacturer, is not guaranteed or endorsed by the publisher.

Copyright $\odot 2022$ Cossiga, Sanduzzi-Zamparelli, Sapena, Guarino, Dallio, Torrisi, Pignata, Federico, Salomone and Morisco. This is an open-access article distributed under the terms of the Creative Commons Attribution License (CC BY). The use, distribution or reproduction in other forums is permitted, provided the original author(s) and the copyright owner(s) are credited and that the original publication in this journal is cited, in accordance with accepted academic practice. No use, distribution or reproduction is permitted which does not comply with these terms. 